\title{
Assessing cardiometabolic risk in middle-aged adults using body mass index and waist-height ratio: are two indices better than one? A cross-sectional study
}

Seán R. Millar ${ }^{*}$, Ivan J. Perry and Catherine M. Phillips

\begin{abstract}
Background: A novel obesity classification method has been proposed using body mass index (BMI) and waistheight ratio (WHtR) together. However, the utility of this approach is unclear. In this study we compare the metabolic profiles in subjects defined as overweight or obese by both measures. We examine a range of metabolic syndrome features, pro-inflammatory cytokines, acute-phase response proteins, coagulation factors and white blood cell counts to determine whether a combination of both indices more accurately identifies individuals at increased obesityrelated cardiometabolic risk.
\end{abstract}

Methods: This was a cross-sectional study involving a random sample of 1856 men and women aged 46-73 years. Metabolic and anthropometric profiles were assessed. Linear and logistic regression analyses were used to compare lipid, lipoprotein, blood pressure, glycaemic and inflammatory biomarker levels between BMI and WHtR tertiles. Multinomial logistic regression was performed to determine cardiometabolic risk feature associations with $\mathrm{BMI}$ and $\mathrm{WHtR}$ groupings. Receiver operating characteristic curve analysis was used to evaluate index discriminatory ability.

Results: The combination of BMI and WHtR tertiles identified consistent metabolic variable differences relative to those characterised on the basis of one index. Similarly, odds ratios of having cardiometabolic risk features were noticeably increased in subjects classified as overweight or obese by both measures when compared to study participants categorised by either BMI or WHtR separately. Significant discriminatory improvement was observed for detecting individual cardiometabolic risk features and adverse biomarker levels. In a fully adjusted model, only individuals within the highest tertile for both indices displayed a significant and positive association with pre-diabetes, OR: 3.4 (95\% Cl: 1.9, 6.0), P $<0.001$.

Conclusions: These data provide evidence that the use of BMI and WHtR together may improve body fat classification. Risk stratification using a composite index may provide a more accurate method for identifying high and low-risk subjects.

Keywords: Body mass index, Waist-height ratio, Cardiometabolic risk, Screening

\section{Background}

Excess body fat has been shown to be associated with dyslipidaemia, hypertension, insulin resistance, chronic

\footnotetext{
*Correspondence: s.millar@ucc.ie

Department of Epidemiology and Public Health, HRB Centre for Health and Diet Research, University College Cork, 4th Floor, Western Gateway Building, Western Road, Cork, Ireland
}

low-grade inflammation and the development of metabolic syndrome (MetS), type 2 diabetes and cardiovascular complications [1-4]. Numerous studies have also demonstrated a high mortality rate in subjects with a body mass index (BMI) $\geq 30 \mathrm{~kg} / \mathrm{m}^{2}[5]$. But because it is a weight-for-height measure, BMI is unable to distinguish between fat and lean mass and elevated BMI may 
not always indicate increased adiposity or predict cardiometabolic events [6, 7].

Evidence suggests that central obesity is a more important metabolic risk factor and waist circumference (WC) measurement has been recommended as a method for central obesity assessment $[8,9]$. However, as WC diagnostic thresholds are different for men and women, and may vary between ethnic groups [10], the practical utility of WC measurement has been questioned [11].

The waist-height ratio (WHtR) (WC divided by height) has been advocated as an alternative surrogate measure of central obesity [12]. As a ratio, this index may circumvent problematic issues relating to gender or populationspecific risk cut-points [13, 14]. But results from studies which have compared BMI and WHtR discriminatory abilities have been inconclusive, with some showing WHtR to be only marginally superior to BMI for predicting cardiometabolic outcomes [15-17].

The prevalence of obesity has escalated in many world populations [18]. Thus, there is an increasing need to identify overweight and obese individuals at highest odds of developing cardiometabolic diseases. Recently, a new obesity classification method was proposed, utilising BMI in conjunction with WHtR [14]. Risk stratification using a composite index may provide a more effective method for identifying high and low-risk subjects. This could allow earlier diagnosis, thus attenuating metabolic complications and chronic morbidity development.

The aim of this study was to compare the metabolic profiles in subjects defined as overweight or obese, using BMI and WHtR, in a random sample of 1856 middleaged men and women. In particular, we examined a range of MetS features, pro-inflammatory cytokines, acutephase response proteins, coagulation factors and white blood cell (WBC) counts to determine whether a combination of BMI and WHtR more accurately identifies individuals at increased obesity-related cardiometabolic risk.

\section{Methods}

\section{Study population}

The Cork and Kerry Diabetes and Heart Disease Study (Phase II) was a single centre, cross-sectional study conducted between 2010 and 2011. A random sample was recruited from a large primary care centre in Mitchelstown, County Cork, Ireland. The Livinghealth Clinic serves a population of approximately 20,000, with a mix of urban and rural residents. Stratified sampling was employed to recruit equal numbers of men and women from all registered attending patients in the 46-73 year age group. In total, 3807 potential participants were selected from the practice list. Following the exclusion of duplicates, deaths, and subjects incapable of consenting or attending appointment, 3051 were invited to participate in the study and of these, 2047 (49.2\% male) completed the questionnaire and physical examination components of the baseline assessment (response rate: $67.1 \%$ ). Details regarding the study design, sampling procedures and methods of data collection have been reported previously [19].

Ethics committee approval conforming to the Declaration of Helsinki was obtained from the Clinical Research Ethics Committee of University College Cork. A letter signed by the contact GP in the clinic was sent out to all selected participants with a reply slip indicating acceptance or refusal. All subjects gave signed informed consent, including permission to use their data for research purposes.

\section{Clinical and laboratory procedures}

All study participants attended the clinic in the morning after an overnight fast and blood samples were taken on arrival. Data on age, gender, morbidity, prescription ( $\mathrm{Rx})$ medication use and smoking and alcohol behaviours were gathered through a self-completed General Health Questionnaire (GHQ). Physical activity levels were assessed using the validated International Physical Activity Questionnaire (IPAQ) [20]. Three independent measurements of systolic and diastolic blood pressure (BP) were obtained with the subject in a seated position using an Omron M7 digital sphygmomanometer (Omron Healthcare Co. Ltd., Japan). The mean of the second and third readings was considered to be a subject's BP.

Triglyceride and high density lipoprotein cholesterol (HDL-C) levels were measured by Cork University Hospital Biochemistry Laboratory on Olympus 5400 biochemistry analysers with Olympus reagents using standardised procedures and fresh samples (Olympus Diagnostica GmbH, Hamburg, Germany). Glucose concentrations were determined using a glucose hexokinase assay (Olympus Life and Material Science Europa Ltd., Lismeehan, Co. Clare, Ireland) and glycated haemoglobin $\mathrm{A}_{1 \mathrm{c}}\left(\mathrm{HbA}_{1 \mathrm{c}}\right)$ levels were measured in the haematology laboratory on an automated high-pressure liquid chromatography instrument Tosoh G7 [Tosoh HLC-723 (G7), Tosoh Europe N.V, Tessenderlo, Belgium]. Serum insulin, c-reactive protein (CRP), tumour necrosis factor alpha (TNF- $\alpha$ ), interleukin 6 (IL-6), adiponectin, leptin, resistin and plasminogen activator inhibitor-1 (PAI-1) were assessed using a biochip array system (Evidence Investigator; Randox Laboratories, UK). Complement component 3 (C3) was measured by immunoturbidimetric assay (RX Daytona; Randox Laboratories). White blood cell counts were determined by flow cytometry technology as part of a full blood count. 


\section{Classification of biochemical and blood pressure measurements}

Patients with type 2 diabetes indicated by either $\mathrm{HbA}_{1 \mathrm{c}}$ levels $\geq 6.5 \%(\geq 48 \mathrm{mmol} / \mathrm{mol})$ or FPG levels $\geq 7.0 \mathrm{mmol} / \mathrm{l}$, a self-reported physician diagnosis, $\mathrm{Rx}$ diabetes medication use, or those who were on insulin therapy, were excluded $(\mathrm{N}=184)$. Lipid, lipoprotein and BP measurements were classified according to National Cholesterol Education Program Adult Treatment Panel III guidelines [21]. Abnormal metabolic risks were defined as high triglycerides $\geq 1.7 \mathrm{mmol} / \mathrm{l}$ and low HDL-C ( $<1.03 \mathrm{mmol} / \mathrm{l}$ in males or $<1.29 \mathrm{mmol} / \mathrm{l}$ in females). Dyslipidaemia was determined according to both high triglyceride and low HDL-C levels. High BP was classified as systolic $\mathrm{BP} \geq 130 \mathrm{mmHg}$ and/or diastolic $\mathrm{BP} \geq 85 \mathrm{mmHg}$ or $\mathrm{Rx}$ anti-hypertensive medication use. The Homeostasis Model Assessment of Insulin Resistance (HOMA-IR) [22] was derived from fasting glucose and insulin concentrations as [(fasting plasma glucose $\mathrm{x}$ fasting serum insulin)/22.5] and insulin resistance was defined as a level equal to or above the 75 th percentile in the study sample. Having three or more MetS risk features ( $\geq 3$ metabolic features) was characterised as any combination of the following: high triglycerides, low HDL-C levels, high BP and insulin resistance. Subjects were classified as having pre-diabetes if they had both elevated $\mathrm{HbA}_{1 \mathrm{c}}$ levels $\geq 5.7 \%(\geq 39 \mathrm{mmol} / \mathrm{mol})$ and impaired fasting plasma glucose levels $\geq 5.6 \mathrm{mmol} / \mathrm{l}$ [23]. As internationally recognised risk cut-points for the examined biomarkers have not been established, we classified inflammation and raised immune activation as a level equal to or above the 75th percentile for each biomarker (C3, CRP, IL-6, TNF- $\alpha$, leptin, resistin, PAI-1 and WBC) with the exception of adiponectin (equal to or below the 25 th percentile).

\section{Anthropometric variables}

The weight and height of each participant were measured to the nearest $0.1 \mathrm{~kg}$ and $0.1 \mathrm{~cm}$ respectively. Portable electronic Tanita WB-100MA weighing scales (Tanita Corporation, IL, USA) were placed on a firm, flat surface and were calibrated weekly to ensure accuracy. Height was measured using a portable Seca Leicester height/ length stadiometer (Seca, Birmingham, UK) and BMI was calculated as weight divided by the square of height. Waist circumference was measured immediately below the lowest rib at the mid-axillary line on bare skin. Subjects were instructed to breathe in, and then out, and to hold their breath while measurement was made to the nearest $0.1 \mathrm{~cm}$ using a Seca 200 measuring tape. The WHtR was calculated as WC divided by height. Two independent readings were taken for $\mathrm{WC}$ and the mean of the two was used in analysis.
Both BMI and WHtR were divided into equal tertiles. Subjects were categorised on the basis of their BMI or WHtR percentiles as normal weight $(<33 \%)$, overweight (33-66 \%) and obese (>66\%). In our sample these cutpoints corresponded to $<26.2,26.2-29.7$, $>29.7$ for BMI and $<0.52,0.52-0.58,>0.58$ for WHtR. The BMI and WHtR groups were combined to form a 5-category variable: (1) normal weight by both, (2) overweight by either, (3) overweight by both, (4) obese by either and (5) obese by both. Overweight subjects classified as obese by either index were assigned to the higher category. Seven subjects had missing anthropometric values and were excluded from statistical analysis.

\section{Lifestyle data}

Lifestyle variables utilised from the IPAQ [20] and GHQ included physical activity level, smoking status and alcohol use. Self-reported physical activity within the previous 6 months was collapsed into two categories: high or moderate $(N=1324)$ and no physical exercise $(N=312)$. Subjects were considered to be current smokers if they smoked cigarettes during the recruitment phase of the study $(N=257)$. Alcohol use was assessed by asking study participants how often they consumed alcohol on a monthly or weekly basis, and was dichotomised as follows: 'never or less than once a month' and '2-4 times monthly'-occasional drinker $(N=1165)$, and 'twice or more weekly'-regular drinker $(N=614)$.

\section{Statistical analysis}

Data analysis was conducted using IBM SPSS Statistics Version 20 (IBM Corp., Armonk, NY, USA) and Stata SE Version 13 (Stata Corporation, College Station, TX, USA) for Windows. Descriptive characteristics were examined according to normal weight, overweight and obese defined by BMI and WHtR tertiles. Dichotomous features are presented as percentages and continuous variables are shown as a mean (plus or minus one standard deviation) or a median and interquartile range for skewed data. Linear and logistic regression (adjusting for gender) were used to examine continuous and dichotomous metabolic variable differences between overweight and obese categories. Skewed continuous data were $\log _{10}$ transformed. Multinomial logistic regression was performed to determine cardiometabolic risk feature associations with each BMI and WHtR tertile combination. Subjects classified as normal weight by both indices were used as the reference category. All multinomial regression models were adjusted using age, gender, physical activity, smoking status and alcohol use as independent covariates.

The discriminatory ability of BMI, WHtR, and both BMI and WHtR used together, was assessed using 
receiver operating characteristic curve (ROC) analysis. The area under the curve (AUC) provides a scale from 0.5 to 1.0 (with 0.5 representing random chance and 1.0 indicating perfect discrimination) by which to appraise the capacity of an obesity index to detect a positive result. Three separate analyses were performed. The first analysis assessed each anthropometric measure as a continuous variable. The second analysis explored cardiometabolic risk feature discrimination using index tertiles. A final analysis examined the 5-category BMI/WHtR combination variable used in previous regression models. Significant differences between AUC values were determined. For all analyses, a P value (two-tailed) of less than 0.05 was considered to indicate statistical significance.

\section{Results}

\section{Descriptive characteristics}

The characteristics of the study population were summarised according to BMI and WHtR tertiles (Table 1). A higher tertile level was related to an increased cardiometabolic risk profile as defined by lipid/lipoprotein, BP, glycaemic indicator and biomarker levels, with obese groups showing the highest proportion of cardiometabolic risk factors. In general, cardiometabolic profiles were broadly similar across BMI and WHtR overweight and obese categories, with the percentage of subjects with dyslipidaemia, high $\mathrm{BP}$, insulin resistance, $\geq 3$ metabolic features and pre-diabetes showing little variation according to classification by either index.

\section{Cardiometabolic profiles according to classification of normal weight, overweight and obese}

The levels of agreement between normal weight, overweight and obese tertiles are shown in Fig. 1. Kappa statistics were similar for normal and obese classifications (Kappa: 0.66, SE: 0.02 for normal weight vs. Kappa: 0.68, SE: 0.02 for obese) with marginal overlap between subjects defined as overweight (Kappa: 0.38, SE: 0.02). In both overweight and obese groups (Table 2), the combination of BMI and WHtR tertiles identified consistent and significant metabolic variable differences relative to those characterised on the basis of one index. Subjects that were classified as overweight or obese by both indices displayed higher mean BMI, WC and median triglyceride levels, reduced HDL-C and adiponectin concentrations, and a higher percentage had adverse biomarker levels, insulin resistance, metabolic feature clustering and pre-diabetes.

\section{Associations between cardiometabolic risk features and BMI/WHtR combinations}

Table 3 presents results from multinomial logistic regression models examining each BMI and WHtR tertile combination. A clear dose-response association was noted, with odds ratios of having cardiometabolic risk features being noticeably increased in subjects classified by both indices. In univariate analysis (not shown), odds ratios of having pre-diabetes were 0.6 (95\% CI: 0.3, 1.5), 1.9 (95 \% CI: 1.1, 3.4), 1.8 (95 \% CI: 1.0, 3.3) and 4.1 (95\% CI: 2.5, 6.7) for subjects categorised as overweight by either, overweight by both, obese by either and obese by both measures respectively. In a fully adjusted model, only patients within the highest BMI and WHtR tertile displayed a significant and positive association with prediabetes defined by both $\mathrm{HbA}_{1 \mathrm{c}}$ and fasting plasma glucose levels, OR: 3.4 (95 \% CI: 1.9, 6.0), P < 0.001 .

\section{Discrimination of cardiometabolic risk features}

In ROC analysis (Table 4), when used as a continuous variable, significantly higher AUC values for WHtR were found to discriminate high triglycerides, $\geq 3$ metabolic features, elevated $\mathrm{C} 3$ and WBC levels when compared to BMI. BMI displayed a significantly higher AUC for detecting increased leptin levels compared to WHtR. A combination of both measures displayed significantly higher discriminatory accuracy for high triglycerides, metabolic feature clustering, C3 and CRP compared to BMI, and for leptin compared to WHtR. Significant improvement for detecting insulin resistance and high WBC levels were noted compared to when either BMI or WHtR were used independently.

When indices were examined as tertiles, significant differences between BMI and WHtR remained for discriminating high triglyceride, leptin and WBC concentrations. The BMI/WHtR 5-category variable was a significantly better discriminator of high triglycerides, low HDL-C, pre-diabetes, high C3, CRP, IL-6, TNF- $\alpha$ and WBC levels compared to BMI, and of leptin compared to WHtR. Significantly higher AUC values for detecting insulin resistance and $\geq 3$ metabolic features were also found compared to when either measure were used alone.

\section{Discussion}

The aim of this study was to determine whether risk stratification using BMI and WHtR together more accurately identifies individuals at increased obesity-related cardiometabolic risk. Our findings indicate that both measures classify different subjects, particularly within the overweight range. These results also demonstrate that individuals defined as overweight or obese, by both $\mathrm{BMI}$ and WHtR, exhibit different cardiometabolic profiles compared to subjects categorised by either index separately. Participants identified by both measures demonstrated stronger associations with individual cardiometabolic risk factors, metabolic feature clustering and displayed a more pro-inflammatory, pro-antherogenic 
Table 1 Characteristics of the study population

\begin{tabular}{|c|c|c|c|c|c|c|}
\hline \multirow[t]{2}{*}{ Feature } & \multicolumn{2}{|l|}{ Normal weight } & \multicolumn{2}{|l|}{ Overweight } & \multicolumn{2}{|l|}{ Obese } \\
\hline & $\mathrm{BMI}(\mathrm{N}=619)$ & WHtR $(\mathrm{N}=619)$ & BMI (N = 618) & WHtR $(\mathrm{N}=618)$ & BMI (N = 619) & WHtR $(\mathrm{N}=619)$ \\
\hline Male & $212(34.2)$ & $145(23.4)$ & $346(56.0)$ & $349(56.5)$ & $327(52.8)$ & $391(63.2)$ \\
\hline Age & $58(54,63)$ & $57(54,62)$ & $59(54,63)$ & $59(54,64)$ & $60(55,64)$ & $60(55,64)$ \\
\hline Weight (kg) & $64.6 \pm 8.5$ & $65.6 \pm 9.4$ & $78.3 \pm 9.1$ & $78.6 \pm 10.2$ & $92.5 \pm 12.6$ & $91.2 \pm 13.5$ \\
\hline BMI $\left(\mathrm{kg} / \mathrm{m}^{2}\right)$ & $23.7 \pm 1.8$ & $24.3 \pm 2.5$ & $27.9 \pm 1.0$ & $27.9 \pm 2.2$ & $33.3 \pm 3.5$ & $32.7 \pm 4.0$ \\
\hline WC $(\mathrm{cm})$ & $79.7 \pm 8.9$ & $77.7 \pm 7.2$ & $91.8 \pm 8.0$ & $92.2 \pm 5.8$ & $102.7 \pm 10.0$ & $104.3 \pm 8.4$ \\
\hline WHtR & $0.48 \pm 0.04$ & $0.47 \pm 0.03$ & $0.55 \pm 0.04$ & $0.55 \pm 0.02$ & $0.62 \pm 0.05$ & $0.62 \pm 0.04$ \\
\hline Triglycerides (mmol/l) & $1.0(0.8,1.3)$ & $1.0(0.8,1.3)$ & $1.2(0.9,1.7)$ & $1.2(0.9,1.7)$ & $1.4(1.0,2.0)$ & $1.5(1.1,2.0)$ \\
\hline High triglycerides $^{a}$ & $67(11.0)$ & $49(8.0)$ & $147(24.5)$ & $152(25.5)$ & 195 (33.3) & $208(35.1)$ \\
\hline HDL-C (mmol/l) & $1.7 \pm 0.4$ & $1.7 \pm 0.4$ & $1.4 \pm 0.3$ & $1.4 \pm 0.3$ & $1.3 \pm 0.3$ & $1.3 \pm 0.3$ \\
\hline Low HDL-C ${ }^{b}$ & $46(7.6)$ & $39(6.4)$ & $74(12.2)$ & $80(13.2)$ & $142(23.8)$ & $143(24.0)$ \\
\hline Dyslipidaemia & $12(2.0)$ & $10(1.6)$ & $37(6.1)$ & $37(6.1)$ & $69(11.5)$ & $71(11.9)$ \\
\hline Systolic BP (mmHg) & $124.9 \pm 17.4$ & $124.8 \pm 17.2$ & $129.5 \pm 15.5$ & $129.2 \pm 15.5$ & $133.0 \pm 15.9$ & $133.4 \pm 16.0$ \\
\hline Diastolic BP (mmHg) & $77.2 \pm 9.6$ & $77.8 \pm 9.6$ & $80.7 \pm 8.8$ & $80.3 \pm 9.4$ & $82.5 \pm 9.9$ & $82.3 \pm 9.5$ \\
\hline High $\mathrm{BP}^{\mathrm{C}}$ & $271(43.8)$ & $263(42.5)$ & $359(58.2)$ & $366(59.3)$ & $471(76.3)$ & $472(76.5)$ \\
\hline Glucose (mmol/l) & $4.8(4.5,5.0)$ & $4.7(4.5,5.0)$ & $4.9(4.6,5.2)$ & $4.9(4.7,5.2)$ & $5.1(4.7,5.4)$ & $5.1(4.8,5.5)$ \\
\hline Insulin ( $\mu \mathrm{U} / \mathrm{ml})$ & $5.3(3.8,7.9)$ & $5.3(3.8,7.9)$ & $8.4(5.7,12.0)$ & $8.3(5.6,12.2)$ & $12.9(8.2,18.4)$ & $12.9(8.4,18.4)$ \\
\hline HOMA-IR & $1.1(0.8,1.7)$ & $1.1(0.8,1.7)$ & $1.8(1.2,2.7)$ & $1.8(1.2,2.7)$ & $2.9(1.8,4.3)$ & $2.9(1.9,4.3)$ \\
\hline Insulin resistance ${ }^{d}$ & $31(5.2)$ & $29(4.9)$ & $121(20.2)$ & $122(20.4)$ & $293(49.4)$ & $294(49.6)$ \\
\hline$\geq 3$ metabolic features & $16(2.6)$ & $13(2.1)$ & $63(10.2)$ & $59(9.5)$ & $163(26.3)$ & $170(27.5)$ \\
\hline $\mathrm{HbA}_{1 c}(\%)$ & $5.6(5.4,4.8)$ & $5.6(5.4,5.8)$ & $5.7(5.5,5.8)$ & $5.7(5.5,5.9)$ & $5.7(5.5,6.0)$ & $5.7(5.5,6.0)$ \\
\hline Pre-diabetes ${ }^{e}$ & $27(4.4)$ & $26(4.3)$ & $49(8.1)$ & $41(6.7)$ & $86(14.2)$ & $95(15.7)$ \\
\hline C3 (mg/dl) & $125.7 \pm 22.9$ & $125.2 \pm 22.4$ & $134.2 \pm 22.7$ & $135.2 \pm 22.8$ & $144.5 \pm 22.9$ & $144.0 \pm 22.8$ \\
\hline $\operatorname{High} C 3^{f}$ & $79(13.2)$ & $77(12.8)$ & $133(22.0)$ & $137(22.6)$ & $239(39.8)$ & $237(39.6)$ \\
\hline $\operatorname{CRP}(\mathrm{ng} / \mathrm{ml})$ & $1.1(0.8,1.6)$ & $1.1(0.8,1.6)$ & $1.3(1.0,1.9)$ & $1.3(1.0,2.0)$ & $1.7(1.2,3.1)$ & $1.8(1.2,3.0)$ \\
\hline High CRP ${ }^{f}$ & $91(15.1)$ & $85(14.1)$ & $124(20.5)$ & $136(22.4)$ & $236(39.4)$ & $230(38.5)$ \\
\hline IL-6 (pg/ml) & $1.4(1.0,2.3)$ & $1.4(1.0,2.1)$ & $1.6(1.2,2.5)$ & $1.7(1.2,2.5)$ & $2.1(1.5,3.3)$ & $2.2(1.5,3.4)$ \\
\hline High IL-6 ${ }^{f}$ & $118(19.5)$ & $102(16.9)$ & $126(20.9)$ & $129(21.3)$ & $207(34.5)$ & $220(36.8)$ \\
\hline TNF- $\alpha(p g / m l)$ & $5.6(4.6,6.9)$ & $5.5(4.5,6.6)$ & $5.9(4.9,7.2)$ & $5.8(4.9,7.1)$ & $6.3(5.2,7.5)$ & $6.4(5.3,7.7)$ \\
\hline High TNF- $\alpha^{f}$ & $117(19.4)$ & $110(18.2)$ & $153(25.4)$ & $140(23.1)$ & $181(30.2)$ & $201(33.6)$ \\
\hline Adiponectin (ng/ml) & $6.6(4.2,9.8)$ & $6.9(4.7,10.2)$ & $4.6(2.9,6.9)$ & $4.6(2.9,6.9)$ & $4.1(2.6,6.3)$ & $3.8(2.5,5.5)$ \\
\hline Low adiponectin ${ }^{f}$ & $78(12.9)$ & $65(10.8)$ & $176(29.1)$ & $167(27.6)$ & 199 (33.2) & $221(36.9)$ \\
\hline Leptin (ng/ml) & $1.3(0.6,2.0)$ & $1.4(0.8,2.1)$ & $1.8(1.0,2.7)$ & $1.8(1.0,2.8)$ & $3.1(1.9,5.1)$ & $2.7(1.6,4.7)$ \\
\hline High leptin ${ }^{f}$ & $39(6.5)$ & $67(11.1)$ & $109(18.0)$ & $122(20.1)$ & $303(50.5)$ & $262(43.7)$ \\
\hline Resistin (ng/ml) & $4.8(3.9,6.4)$ & $4.9(3.8,6.4)$ & $4.9(3.7,6.5)$ & $4.9(3.8,6.6)$ & $5.3(4.0,7.0)$ & $5.2(4.0,6.7)$ \\
\hline High resistin ${ }^{f}$ & $133(22.0)$ & $136(22.5)$ & $141(23.3)$ & $152(25.1)$ & $178(29.7)$ & $164(27.4)$ \\
\hline PAl-1 (ng/ml) & $24.3 \pm 10.3$ & $24.0 \pm 10.4$ & $28.1 \pm 13.6$ & $27.5 \pm 11.7$ & $28.7 \pm 12.0$ & $29.7 \pm 13.7$ \\
\hline High PAl- $1^{f}$ & $100(16.6)$ & $94(15.6)$ & $164(27.2)$ & $161(26.6)$ & $187(31.2)$ & $196(32.8)$ \\
\hline WBC $\left(10^{9} / \mathrm{l}\right)$ & $5.7 \pm 2.4$ & $5.5 \pm 1.6$ & $5.8 \pm 1.6$ & $5.9 \pm 2.3$ & $6.1 \pm 1.5$ & $6.3 \pm 1.5$ \\
\hline High WBC & $125(20.7)$ & $103(17.0)$ & $149(24.5)$ & $153(25.3)$ & $177(29.7)$ & $195(32.7)$ \\
\hline \multicolumn{7}{|c|}{$\begin{array}{l}\text { Mean and } \pm \text { standard deviation are shown for continuous variables. Age, triglycerides, glucose, insulin, HOMA-IR, HbA, } \mathrm{CRP}_{1}, \mathrm{LL}-6, \mathrm{TNF}-\alpha, \text { adiponectin, leptin and } \\
\text { resistin are shown as a median (interquartile range). } \% \text { (in brackets) for dichotomous variables will vary as some variables have missing values }\end{array}$} \\
\hline \multicolumn{7}{|l|}{ a Triglycerides $\geq 1.7$} \\
\hline \multicolumn{7}{|c|}{ b $\mathrm{HDL}-\mathrm{C}<1.03$ (males) or HDL-C $<1.29$ (females) } \\
\hline \multicolumn{7}{|c|}{ c Systolic BP $\geq 130$ and/or diastolic BP $\geq 85$ or use of $\mathrm{Rx}$ anti-hypertensives } \\
\hline \multicolumn{7}{|l|}{${ }^{d}$ HOMA-IR $\geq 2.96$} \\
\hline \multicolumn{7}{|c|}{ e Both $\mathrm{HbA}_{1 \mathrm{c}}$ levels $\geq 5.7 \%$ and fasting plasma glucose levels $\geq 5.6$} \\
\hline f Threshold: C3 $\geq 148 ;$ CRP & $2.25 ; \mathrm{IL}-6 \geq 2.72 ; \mathrm{TNF}$ & $\geq 7.2 ;$ adiponectin $\leq$ & $\operatorname{tin} \geq 3.07 ;$ resi & 6; & & \\
\hline
\end{tabular}




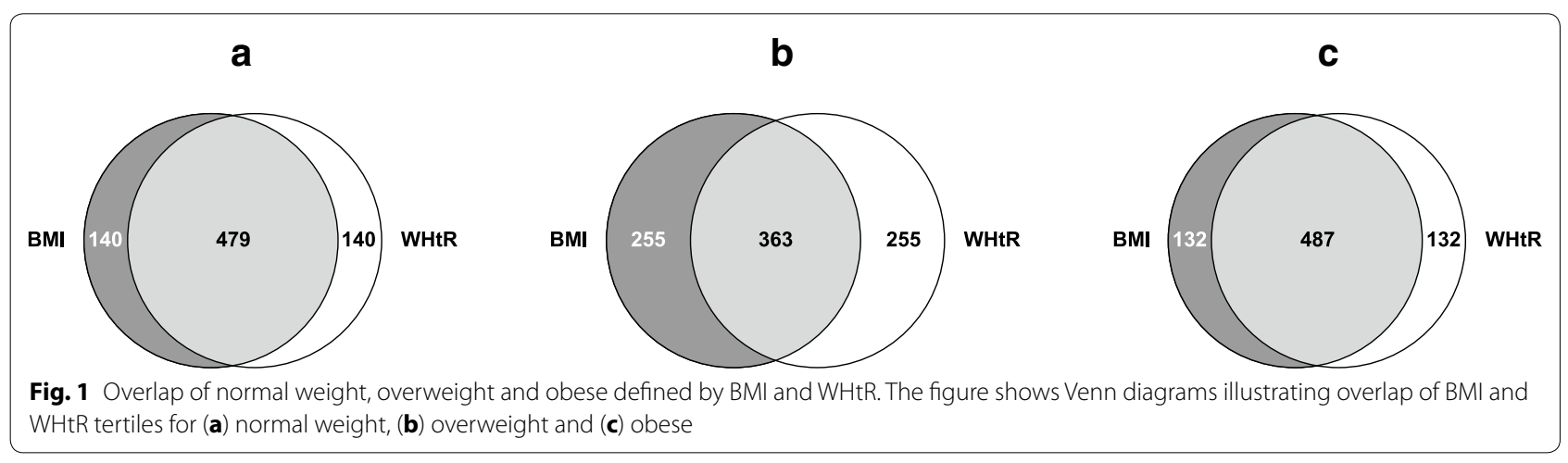

and insulin resistant profile. Use of both indices also significantly improved discrimination of cardiometabolic risk features. These results suggest that joint use of BMI and WHtR may be clinically useful as a method to detect individuals at risk of cardiometabolic disorders.

Although it is straightforward to assess, and easy to calculate, limitations regarding the use of BMI as a sole method for adiposity appraisal have been widely acknowledged [7, 11]. Though frequently employed within epidemiological research and healthcare practice, BMI does not discriminate between fat and lean body mass, therefore persons of short stature or muscular build may be misidentified [24]. Recent research has indicated that general obesity categorisation based on BMI might be inadequate [25]. Importantly, the finding that approximately half of obese subjects are metabolically healthy when classified using dual-energy X-ray absorptiometry-derived body fat percentage, compared to approximately one-third by BMI [26], signals that caution should be exercised with regard to how obesity is defined [24].

Compared with BMI, WC is thought to be more strongly correlated with visceral adipose tissue (VAT) which has been shown to be associated with increased risk of dyslipidaemia, hypertension and type 2 diabetes $[9,27,28]$. Though the exact mechanism of association between VAT and metabolic risk is still poorly understood, research has implied that fatty acids released from VAT drain into the liver and skeletal muscle, causing metabolic dysfunction within these organs [29]. Adipokines secreted from VAT may also contribute to cardiometabolic disease through inflammation of vascular tissue $[8,30]$. Although WC measurement has been recommended as a method for VAT and cardiometabolic risk assessment, controversy exists regarding its clinical efficacy. In particular, the need for gender and ethnicspecific risk cut-points, and the fact that WC does not take whole body fat distribution into account, indicate constraints regarding its practical application and usefulness within a clinical setting [11].
The WHtR is potentially advantageous as it may not require conversion to gender or population-specific cut-offs or percentiles [13]. It has been previously suggested that a WHtR $\geq 0.5$ may serve as a useful boundary for increased cardiometabolic risk, with a WHtR $\geq 0.6$ threshold indicating substantially increased risk [14]. Additionally, it has been shown that height has an inverse association with cardiovascular disease mortality and total mortality [31, 32], indicating that its use within an adiposity variable may be clinically important. In a recent meta-analysis of 31 prospective or cross-sectional studies, Ashwell et al. demonstrated WHtR to be a better discriminator of hypertension, metabolic syndrome, type 2 diabetes and cardiovascular disease when compared to BMI [15]. Pooled results showed that WHtR improved discrimination of all outcomes by $3-4 \%$. However, other studies have suggested that differences in predictive abilities are minimal, and have questioned the measurement of height in addition to WC [17].

The results from our research suggest that both BMI and WHtR provide important and independent information, and that joint measurement may help refine body fat risk classification. Within our sample it was noted that participants who were categorised as overweight on the basis of one index also displayed an increased cardiometabolic risk profile. As a percentage of these individuals might be considered normal weight if either measure were used alone, these results indicate that use of both indices may provide a more sensitive method for detecting patients at increased cardiometabolic risk. We also observed noticeably strong associations with cardiometabolic risk factors in subjects who were classified as overweight or obese by both BMI and WHtR together. This suggests that joint measurement may equally provide a more specific procedure for identifying high-risk subjects within overweight and obese categories. In particular, patients within the highest tertile for both indices were at a significantly higher risk compared to other obese subjects. In addition, a significant association with pre-diabetes was only observed within this tertile after 
Table 2 Cardiometabolic profiles according to classification of normal weight, overweight and obese defined by either BMI, WHtR or both

\begin{tabular}{|c|c|c|c|c|c|c|c|}
\hline Feature & $\begin{array}{l}\text { Normal weight } \\
\text { by both }(N=479)\end{array}$ & $\begin{array}{l}\text { Overweight } \\
\text { by either }(N=263)\end{array}$ & $\begin{array}{l}\text { Overweight } \\
\text { by both }(\mathrm{N}=363)\end{array}$ & Pvalue $^{a}$ & $\begin{array}{l}\text { Obese by either } \\
(\mathrm{N}=264)\end{array}$ & $\begin{array}{l}\text { Obese by both } \\
(N=487)\end{array}$ & $P_{\text {value }}$ \\
\hline Male $e^{c}$ & $122(25.5)$ & $105(39.9)$ & $231(63.6)$ & $<0.001$ & $136(51.5)$ & $291(59.8)$ & 0.03 \\
\hline $\operatorname{Age}^{d}$ & $58(54,62)$ & $58(54,65)$ & $59(54,63)$ & 0.885 & $59(54,64)$ & $60(55,64)$ & 0.15 \\
\hline Weight $(\mathrm{kg})^{\mathrm{e}}$ & $63.1 \pm 8.1$ & $71.9 \pm 8.0$ & $79.6 \pm 9.2$ & $<0.001$ & $81.8 \pm 9.5$ & $94.6 \pm 12.6$ & $<0.001$ \\
\hline BMI $\left(\mathrm{kg} / \mathrm{m}^{2}\right)^{\mathrm{e}}$ & $23.3 \pm 1.8$ & $26.2 \pm 1.5$ & $27.9 \pm 1.0$ & $<0.001$ & $29.7 \pm 1.9$ & $33.8 \pm 3.6$ & $<0.001$ \\
\hline$W C(\mathrm{~cm})^{\mathrm{e}}$ & $76.6 \pm 7.2$ & $85.7 \pm 6.7$ & $93.0 \pm 5.7$ & $<0.001$ & $95.1 \pm 7.2$ & $105.7 \pm 8.5$ & $<0.001$ \\
\hline WHtRe & $0.46 \pm 0.03$ & $0.52 \pm 0.03$ & $0.55 \pm 0.02$ & $<0.001$ & $0.57 \pm 0.03$ & $0.63 \pm 0.05$ & $<0.001$ \\
\hline $\begin{array}{c}\text { Triglycerides } \\
(\mathrm{mmol} / \mathrm{l})^{\mathrm{e}}\end{array}$ & $0.9(0.7,1.2)$ & $1.1(0.8,1.5)$ & $1.3(0.9,1.7)$ & 0.005 & $1.3(1.0,1.8)$ & $1.5(1.1,2.0)$ & 0.034 \\
\hline High triglycerides ${ }^{\mathrm{e}}$ & $36(7.6)$ & $43(16.7)$ & $93(26.6)$ & 0.031 & $71(27.7)$ & $166(35.8)$ & 0.06 \\
\hline $\mathrm{HDL}-\mathrm{C}(\mathrm{mmol} / \mathrm{l})^{\mathrm{e}}$ & $1.7 \pm 0.4$ & $1.6 \pm 0.4$ & $1.4 \pm 0.3$ & $<0.001$ & $1.4 \pm 0.3$ & $1.3 \pm 0.3$ & $<0.001$ \\
\hline Low HDL-C & $31(6.7)$ & $23(8.8)$ & $46(12.9)$ & 0.038 & $39(15.1)$ & $123(26.3)$ & 0.001 \\
\hline Dyslipidaemia $^{e}$ & $7(1.5)$ & $8(3.1)$ & $21(5.9)$ & 0.059 & $24(9.3)$ & $58(12.3)$ & 0.253 \\
\hline Systolic BP $(\mathrm{mmHg})^{e}$ & $124.2 \pm 17.5$ & $126.4 \pm 16.2$ & $129.4 \pm 14.7$ & 0.027 & $132.5 \pm 16.4$ & $133.4 \pm 15.8$ & 0.52 \\
\hline Diastolic BP $(\mathrm{mmHg})^{\mathrm{e}}$ & $77.1 \pm 9.6$ & $78.8 \pm 9.4$ & $80.5 \pm 8.8$ & 0.003 & $82.2 \pm 9.3$ & $82.5 \pm 9.8$ & 0.61 \\
\hline High BPe & $195(40.7)$ & $134(51.0)$ & $213(58.8)$ & 0.069 & $175(66.3)$ & $384(79.2)$ & $<0.001$ \\
\hline Glucose $(\mathrm{mmol} / \mathrm{l})^{\mathrm{e}}$ & $4.7(4.5,5.0)$ & $4.8(4.6,5.1)$ & $4.9(4.7,5.3)$ & 0.038 & $4.9(4.7,5.2)$ & $5.1(4.8,5.5)$ & $<0.001$ \\
\hline Insulin $(\mu \mathrm{U} / \mathrm{ml})^{\mathrm{e}}$ & $5.1(3.7,7.5)$ & $6.2(4.3,9.2)$ & $8.8(6.0,12.1)$ & $<0.001$ & $10.2(6.8,14.3)$ & $14.0(9.0,20.2)$ & $<0.001$ \\
\hline HOMA-IR & $1.1(0.8,1.6)$ & $1.4(0.9,2.0)$ & $2.0(1.3,2.7)$ & $<0.001$ & $2.2(1.5,3.2)$ & $3.2(2.0,4.6)$ & $<0.001$ \\
\hline Insulin resistance $\mathrm{e}^{\mathrm{e}}$ & $20(4.4)$ & $20(7.8)$ & $72(20.5)$ & $<0.001$ & $79(31.1)$ & $254(54.5)$ & $<0.001$ \\
\hline $\begin{array}{l}\geq 3 \text { metabolic }^{\text {features }}{ }^{\text {e }} \\
\text { fol }\end{array}$ & $8(1.7)$ & $13(4.9)$ & $35(9.6)$ & 0.024 & $39(14.8)$ & $147(30.2)$ & $<0.001$ \\
\hline $\mathrm{HbA}_{1 \mathrm{c}}(\%)^{\mathrm{e}}$ & $5.6(5.4,5.8)$ & $5.7(5.5,5.9)$ & $5.7(5.4,5.8)$ & 0.54 & $5.7(5.5,5.8)$ & $5.8(5.6,6.0)$ & 0.002 \\
\hline Pre-diabetes ${ }^{\mathrm{e}}$ & $22(4.7)$ & $8(3.1)$ & $31(8.7)$ & 0.018 & $21(8.1)$ & $80(16.8)$ & 0.002 \\
\hline $\mathrm{C} 3(\mathrm{mg} / \mathrm{dll})^{\mathrm{e}}$ & $124.0 \pm 23.1$ & $130.2 \pm 20.4$ & $134.7 \pm 23.0$ & $<0.001$ & $139.2 \pm 23.2$ & $145.7 \pm 22.9$ & $<0.001$ \\
\hline High $\mathrm{C}^{\mathrm{e}}$ & $56(12.1)$ & $42(16.2)$ & $77(21.8)$ & 0.013 & $76(29.1)$ & $200(42.6)$ & $<0.001$ \\
\hline $\operatorname{CRP}(\mathrm{ng} / \mathrm{ml})^{\mathrm{e}}$ & $1.0(0.8,1.5)$ & $1.3(1.0,2.0)$ & $1.3(1.0,1.8)$ & 0.976 & $1.5(1.1,2.5)$ & $1.8(1.2,3.2)$ & $<0.001$ \\
\hline High CRPe & $60(12.9)$ & $52(20.1)$ & $67(18.9)$ & 0.891 & $78(30.1)$ & $194(41.4)$ & $<0.001$ \\
\hline $\mathrm{IL}-6(\mathrm{pg} / \mathrm{ml})^{\mathrm{e}}$ & $1.3(1.0,2.1)$ & $1.4(1.1,2.4)$ & $1.7(1.2,2.4)$ & 0.317 & $1.9(1.3,2.9)$ & $2.3(1.6,3.5)$ & 0.001 \\
\hline High IL-6 ${ }^{e}$ & $82(17.6)$ & 49 (18.9) & 70 (19.7) & 0.99 & $73(28.3)$ & $177(37.7)$ & 0.01 \\
\hline TNF- $\alpha(\mathrm{pg} / \mathrm{ml})^{\mathrm{e}}$ & $5.5(4.6,6.7)$ & $5.8(4.6,6.9)$ & $5.9(4.9,7.2)$ & 0.521 & $5.8(5.0,7.3)$ & $6.4(5.3,7.0)$ & 0.067 \\
\hline High TNF- $\alpha^{e}$ & $87(18.7)$ & $50(19.4)$ & $89(25.1)$ & 0.276 & $68(26.4)$ & $157(33.4)$ & 0.047 \\
\hline Adiponectin $(\mathrm{ng} / \mathrm{ml})^{\mathrm{e}}$ & $7.0(4.7,10.3)$ & $5.9(3.8,9.0)$ & $4.2(2.7,6.3)$ & $<0.001$ & $4.8(2.9,6.7)$ & $3.8(2.5,5.5)$ & 0.023 \\
\hline Low adiponectin ${ }^{e}$ & $48(10.3)$ & $43(16.6)$ & $117(33.0)$ & 0.007 & $70(27.0)$ & $175(37.2)$ & 0.032 \\
\hline Leptin $(\mathrm{ng} / \mathrm{ml})^{\mathrm{e}}$ & $1.3(0.7,2.0)$ & $1.6(1.0,2.4)$ & $1.7(0.9,2.7)$ & 0.001 & $2.3(1.3,4.0)$ & $3.2(1.9,5.2)$ & $<0.001$ \\
\hline High leptin ${ }^{e}$ & $30(6.5)$ & $39(15.1)$ & $56(15.8)$ & 0.009 & 87 (33.6) & $239(50.9)$ & $<0.001$ \\
\hline Resistin $(\mathrm{ng} / \mathrm{ml})^{\mathrm{e}}$ & $4.9(3.8,6.4)$ & $4.8(3.8,6.7)$ & $4.9(3.7,6.4)$ & 0.946 & $4.9(4.0,6.8)$ & $5.3(4.0,7.0)$ & 0.274 \\
\hline High resistin ${ }^{e}$ & $100(21.5)$ & $66(25.5)$ & $79(22.3)$ & 0.452 & $72(27.9)$ & $135(28.7)$ & 0.611 \\
\hline PAl-1 $(\mathrm{ng} / \mathrm{ml})^{\mathrm{e}}$ & $23.8 \pm 10.2$ & $25.5 \pm 10.9$ & $28.1 \pm 12.0$ & 0.033 & $29.3 \pm 15.6$ & $29.2 \pm 12.1$ & 0.761 \\
\hline High PA1-1 ${ }^{e}$ & $69(14.8)$ & $52(20.2)$ & $103(29.0)$ & 0.046 & $71(27.5)$ & $156(33.2)$ & 0.161 \\
\hline WBC $\left(10^{9} / /\right)^{\mathrm{e}}$ & $5.5 \pm 1.7$ & $5.8 \pm 3.0$ & $5.9 \pm 1.6$ & 0.92 & $6.1 \pm 1.6$ & $6.2 \pm 1.4$ & 0.345 \\
\hline High WBC ${ }^{e}$ & $84(17.9)$ & $56(22.0)$ & $88(24.6)$ & 0.832 & 74 (28.7) & 149 (31.9) & 0.418 \\
\hline
\end{tabular}

Mean and \pm standard deviation are shown for continuous variables. Age, triglycerides, glucose, insulin, HOMA-IR, HbA $1{ }_{1}, \mathrm{CRP}$, IL-6, TNF- $\alpha$, adiponectin, leptin and resistin are shown as a median (interquartile range). \% (in brackets) for dichotomous variables will vary as some variables have missing values

a $\mathrm{P}$ value for difference: overweight by either compared to overweight by both

b $P$ value for difference: obese by either compared to obese by both

c $x^{2}$ for difference

d Mann Whitney U for difference

e $P$ value for difference adjusted for gender. Overweight subjects classified as obese by either index were assigned to the higher category 
Table 3 Odds ratios $(95 \% \mathrm{Cl})$ of having cardiometabolic risk features according to classification of overweight and obese

\begin{tabular}{|c|c|c|c|c|c|c|c|c|}
\hline \multirow[t]{3}{*}{ Feature } & \multicolumn{8}{|c|}{ Odds ratios $(95 \% \mathrm{Cl})^{\mathrm{a}}$} \\
\hline & \multicolumn{4}{|c|}{ Overweight compared to normal weight } & \multicolumn{4}{|c|}{ Obese compared to normal weight } \\
\hline & $\begin{array}{l}\text { Either BMI } \\
\text { or WHtR }\end{array}$ & $P$ value & $\begin{array}{l}\text { Both BMI } \\
\text { and WHtR }\end{array}$ & $P$ value & $\begin{array}{l}\text { Either BMI } \\
\text { or WHtR }\end{array}$ & $P$ value & $\begin{array}{l}\text { Both BMI } \\
\text { and WHtR }\end{array}$ & $P$ value \\
\hline High triglycerides & $2.1(1.3,3.5)$ & 0.003 & $3.5(2.3,5.4)$ & $<0.001$ & $3.4(2.1,5.5)$ & $<0.001$ & $5.6(3.7,8.6)$ & $<0.001$ \\
\hline Low HDL-C & $1.4(0.8,2.5)$ & 0.3 & $2.1(1.3,3.7)$ & 0.005 & $2.2(1.2,3.8)$ & 0.008 & $5.8(3.6,9.2)$ & $<0.001$ \\
\hline Dyslipidaemia & $1.8(0.6,5.3)$ & 0.263 & $3.8(1.5,9.3)$ & 0.004 & $4.6(1.9,11.6)$ & 0.001 & $8.6(3.7,19.6)$ & $<0.001$ \\
\hline High BP & $1.5(1.1,2.1)$ & 0.02 & $2.1(1.5,2.8)$ & $<0.001$ & $3.0(2.1,4.2)$ & $<0.001$ & $5.7(4.1,7.9)$ & $<0.001$ \\
\hline Insulin resistance & $1.8(0.9,3.7)$ & 0.083 & $5.4(3.1,9.6)$ & $<0.001$ & $9.5(5.3,16.8)$ & $<0.001$ & $26.6(15.5,45.7)$ & $<0.001$ \\
\hline $\begin{array}{l}\geq 3 \text { metabolic } \\
\text { features }\end{array}$ & $2.6(1.0,6.6)$ & 0.043 & $5.4(2.4,12.0)$ & $<0.001$ & $7.8(3.5,17.5)$ & $<0.001$ & $22.2(10.5,47.0)$ & $<0.001$ \\
\hline Pre-diabetes & $0.6(0.2,1.4)$ & 0.227 & $1.6(0.9,3.1)$ & 0.142 & $1.6(0.8,3.2)$ & 0.218 & $3.4(1.9,6.0)$ & $<0.001$ \\
\hline High CRP & $1.6(1.0,2.6)$ & 0.032 & $1.8(1.2,2.7)$ & 0.007 & $3.6(2.4,5.5)$ & $<0.001$ & $6.1(4.2,8.9)$ & $<0.001$ \\
\hline High IL-6 & $1.0(0.7,1.6)$ & 0.897 & $1.1(0.8,1.7)$ & 0.541 & $1.7(1.2,2.6)$ & 0.008 & $2.7(1.9,3.8)$ & $<0.001$ \\
\hline High TNF- $\alpha$ & $1.1(0.7,1.6)$ & 0.738 & $1.4(1.0,2.0)$ & 0.089 & $1.2(0.8,1.9)$ & 0.323 & $2.2(1.5,3.1)$ & $<0.001$ \\
\hline Low adiponectin & $1.4(0.8,2.3)$ & 0.204 & $2.7(1.8,4.2)$ & $<0.001$ & $2.6(1.6,4.2)$ & $<0.001$ & $3.9(2.6,6.0)$ & $<0.001$ \\
\hline High leptin & $3.6(2.1,6.3)$ & $<0.001$ & $5.9(3.5,9.9)$ & $<0.001$ & $15.7(9.3,26.6)$ & $<0.001$ & $46.6(27.9,77.6)$ & $<0.001$ \\
\hline High resistin & $1.3(0.9,1.9)$ & 0.205 & $1.3(0.9,1.9)$ & 0.194 & $1.5(1.0,2.2)$ & 0.046 & $1.8(1.2,2.5)$ & 0.001 \\
\hline High PAI-1 & $1.2(0.8,1.8)$ & 0.460 & $2.0(1.3,2.9)$ & $<0.001$ & $1.9(1.3,2.9)$ & 0.002 & $2.7(1.9,3.9)$ & $<0.001$ \\
\hline High WBC & $1.5(1.0,2.4)$ & 0.073 & $1.9(1.2,2.9)$ & 0.003 & $2.7(1.7,4.2)$ & $<0.001$ & $3.2(2.2,4.8)$ & $<0.001$ \\
\hline
\end{tabular}

a Multinomial logistic regression, reference category: normal weight by both BMI and WHtR. Overweight subjects classified as obese by either index were assigned to the higher category. All models adjusted for age, gender, physical activity, smoking and alcohol use

adjustment for other risk factors. This might imply that the relationship between obesity and diabetes is better indicated at this mode and level of adiposity.

Nevertheless, it was also noted that subjects classified by both indices were, on average, more overweight or obese, and thus would probably be identified if either BMI or WHtR were used alone. Additionally, discriminatory improvements for detecting individual cardiometabolic features were modest. However, cardiometabolic diseases are multifactorial, as it has been shown that subjects with a combination of features are at higher risk of cardiometabolic events [33, 34]. Also, a degree of measurement error is to be expected during any anthropometric assessment. We have recently shown that using BMI and WHtR together significantly improves discrimination of type 2 diabetes [30]. Cut-points on the ROC demonstrated greater sensitivity and specificity than when either index were used independently. As the sum of risk factors may be greater than the individual parts for predicting cardiometabolic events, and as measurement error may limit the minimal detectable difference in a cardiometabolic risk parameter [35], it could be that these findings are due to the greater measurement accuracy that joint BMI and WHtR assessment may provide.

Although it is hoped that public health programs may eventually reduce the prevalence of obesity-related metabolic disorders, current strategies to combat obesity are failing as overweight and obesity rates continue to increase worldwide [18]. As a percentage of obese subjects are considered to be metabolically healthy [24], there is an increasing need for cheap and non-invasive methods to detect overweight and obese individuals at highest odds of developing cardiometabolic diseases. In previous research we have shown that assessing both bioelectrical impedance-derived body fat percentage and BMI may help to discriminate individuals at greater cardiometabolic risk than BMI alone [36]. Those identified using both tools had a more metabolically unhealthy profile and were non-responsive to dietary changes. These findings suggest that stratification of obese individuals, based on their metabolic health phenotype, could be important in the early identification of those who should be prioritised for pharmacological and lifestyle interventions [24]. Joint use of BMI and WHtR may provide a convenient and inexpensive means for risk stratification. Such a method might be useful in resource-poor settings where blood sampling is unavailable, or in populations without regular access to primary health services.

As far as we are aware, our study is the first to comprehensively examine the joint use of BMI and WHtR in a middle-aged European population. Strengths include a high participation rate, the use of questionnaires to assess 
Table 4 Area under the receiver operating characteristic curve values $(95 \% \mathrm{Cl})$ for index models to discriminate cardiometabolic risk features

\begin{tabular}{|c|c|c|c|c|c|c|c|c|c|c|c|c|}
\hline \multirow[t]{3}{*}{ Feature } & \multicolumn{6}{|c|}{ As a continuous variable } & \multicolumn{4}{|c|}{ As a categorical variable (tertiles) } & \multirow{2}{*}{\multicolumn{2}{|c|}{$\begin{array}{l}\text { Overweight } \\
\text { and obese } \\
\text { by either or both }\end{array}$}} \\
\hline & \multicolumn{2}{|c|}{ BMI alone } & \multicolumn{2}{|c|}{ WHtR alone } & \multicolumn{2}{|c|}{$\begin{array}{l}\text { Both BMI } \\
\text { and WHtR }\end{array}$} & \multicolumn{2}{|c|}{ BMI alone } & \multicolumn{2}{|c|}{ WHtR alone } & & \\
\hline & AUC & $95 \% \mathrm{Cl}$ & AUC & $95 \% \mathrm{Cl}$ & AUC & $95 \% \mathrm{Cl}$ & AUC & $95 \% \mathrm{Cl}$ & AUC & $95 \% \mathrm{Cl}$ & AUC & $95 \% \mathrm{Cl}$ \\
\hline High triglycerides & 0.68 & $0.65,0.71$ & $0.71^{b}$ & $0.68,0.73$ & $0.71^{b}$ & $0.68,0.74$ & 0.68 & $0.65,0.71$ & $0.70^{d}$ & $0.67,0.73$ & $0.70^{d}$ & $0.67,0.73$ \\
\hline Low HDL-C & 0.67 & $0.63,0.70$ & 0.68 & $0.65,0.72$ & 0.68 & $0.65,0.71$ & 0.66 & $0.62,0.69$ & 0.67 & $0.64,0.71$ & $0.68^{d}$ & $0.64,0.71$ \\
\hline Dyslipidaemia & 0.68 & $0.64,0.73$ & 0.70 & $0.65,0.74$ & 0.70 & $0.65,0.74$ & 0.69 & $0.64,0.73$ & 0.69 & $0.65,0.73$ & 0.70 & $0.66,0.74$ \\
\hline High BP & 0.70 & $0.67,0.72$ & 0.70 & $0.67,0.72$ & 0.70 & $0.68,0.72$ & 0.69 & $0.67,0.72$ & 0.69 & $0.67,0.71$ & 0.70 & $0.67,0.72$ \\
\hline Insulin resistance & 0.80 & $0.78,0.82$ & 0.80 & $0.78,0.83$ & $0.81^{b, c}$ & $0.79,0.83$ & 0.78 & $0.76,0.80$ & 0.77 & $0.75,0.80$ & $0.79^{\mathrm{d}, \mathrm{e}}$ & $0.77,0.82$ \\
\hline$\geq 3$ metabolic features & 0.76 & $0.73,0.79$ & $0.78^{b}$ & $0.75,0.81$ & $0.78^{b}$ & $0.75,0.81$ & 0.75 & $0.72,0.78$ & 0.75 & $0.72,0.78$ & $0.77^{d, e}$ & $0.74,0.80$ \\
\hline Pre-diabetes & 0.70 & $0.66,0.74$ & 0.70 & $0.66,0.74$ & 0.70 & $0.66,0.74$ & 0.67 & $0.63,0.71$ & 0.68 & $0.64,0.72$ & $0.69^{d}$ & $0.65,0.73$ \\
\hline High C3 & 0.70 & $0.67,0.73$ & $0.72^{\mathrm{b}}$ & $0.69,0.75$ & $0.72^{b}$ & $0.69,0.75$ & 0.69 & $0.66,0.71$ & 0.70 & $0.67,0.73$ & $0.71^{d}$ & $0.68,0.73$ \\
\hline High CRP & 0.69 & $0.66,0.72$ & 0.69 & $0.67,0.72$ & $0.70^{b}$ & $0.67,0.72$ & 0.67 & $0.64,0.70$ & 0.68 & $0.65,0.71$ & $0.69^{d}$ & $0.66,0.72$ \\
\hline High IL-6 & 0.66 & $0.63,0.69$ & 0.67 & $0.64,0.69$ & 0.67 & $0.64,0.69$ & 0.65 & $0.62,0.68$ & 0.66 & $0.63,0.69$ & $0.66^{d}$ & $0.63,0.69$ \\
\hline High TNF- $\alpha$ & 0.63 & $0.60,0.66$ & 0.63 & $0.60,0.66$ & 0.63 & $0.60,0.66$ & 0.62 & $0.59,0.65$ & 0.63 & $0.61,0.66$ & $0.63^{d}$ & $0.60,0.66$ \\
\hline Low adiponectin & 0.79 & $0.77,0.81$ & 0.79 & $0.77,0.81$ & 0.79 & $0.77,0.81$ & 0.79 & $0.77,0.81$ & 0.79 & $0.76,0.81$ & 0.79 & $0.77,0.81$ \\
\hline High leptin & $0.86^{\mathrm{C}}$ & $0.84,0.87$ & 0.84 & $0.82,0.86$ & $0.86^{c}$ & $0.84,0.88$ & $0.84^{e}$ & $0.82,0.86$ & 0.81 & $0.79,0.83$ & $0.84^{e}$ & $0.82,0.86$ \\
\hline High resistin & 0.57 & $0.54,0.60$ & 0.56 & $0.53,0.59$ & 0.57 & $0.54,0.60$ & 0.57 & $0.54,0.60$ & 0.56 & $0.53,0.59$ & 0.56 & $0.53,0.60$ \\
\hline High PAI-1 & 0.61 & $0.58,0.64$ & 0.62 & $0.59,0.65$ & 0.62 & $0.59,0.65$ & 0.62 & $0.59,0.64$ & 0.62 & $0.59,0.65$ & 0.62 & $0.59,0.65$ \\
\hline High WBC & 0.59 & $0.56,0.62$ & $0.61^{b}$ & $0.58,0.64$ & $0.63^{b, c}$ & $0.60,0.66$ & 0.58 & $0.55,0.61$ & $0.60^{d}$ & $0.57,0.63$ & $0.59^{d}$ & $0.56,0.62$ \\
\hline
\end{tabular}

All models include age and gender

a 5 -category variable: 1 normal weight by both, 2 overweight by either, 3 overweight by both, 4 obese by either and 5 obese by both. Overweight subjects classified as obese by either index were assigned to the higher category

b $\mathrm{P}<0.05$ compared to BMI (continuous)

c $\mathrm{P}<0.05$ compared to WHtR (continuous)

d $\mathrm{P}<0.05$ compared to BMI (categorical)

e $\mathrm{P}<0.05$ compared to WHtR (categorical)

lifestyle behaviours and inclusion of a wide range of metabolic variables to define cardiometabolic health. Our findings are of potential public health and clinical significance in terms of screening and the use of stratification based on obesity assessment as a method for determining cardiometabolic risk.

Notwithstanding these strengths, methodological limitations should be considered when examining results from this study. Given the modest number of outcomes within our sample, we did not stratify by gender in analysis. Although some studies have implied heterogeneous relationships between measures of adiposity and cardiometabolic outcomes relating to gender [11], previous work by our group has suggested that these may be explained by sex differences in obesity prevalence [30]. In addition, recommended risk cut-points for BMI and WHtR are the same for men and women, and the gender variable was accounted for in statistical analyses.

Also of concern is that we did not use established obesity index cut-offs and that our data were cross-sectional, as this precludes examination of temporal relationships.
Although World Health Organisation cut-points for BMI are commonly used [5], and thresholds for WHtR have been recommended [14, 37], for the purposes of this research it was necessary to place both variables on the same scale. Future studies, utilising longitudinal data, will be needed to evaluate the applicability, validity and reliability of joint measurement [14] using established and recommended diagnostic cut-points. In particular, it will be necessary to determine whether risk stratification, using both BMI and WHtR, is clinically useful and superior to currently recommended BMI classification [38].

Finally, our data were derived from a single primary care based sample which may not be representative of the source population. However, Ireland presents a generally ethnically homogeneous group [39]. Thus, the relationships we observed are likely to be similar in other middle-aged Irish adults. In addition, random sampling of subjects and the use of validated methods for data collection ensured internal sample validity and the results from this research may be generalisable to a similar middleaged Caucasian-European population. 


\section{Conclusions}

In summary, our findings reveal that cardiometabolic risk profiles in individuals defined as overweight or obese, by both BMI and WHtR, are significantly increased when compared to subjects categorised by either index separately. Use of both measures also improved discrimination of individual cardiometabolic risk factors and identified a subset of at-risk individuals who might otherwise be missed. Although assessment of WC, in addition to BMI, competes for the limited time available during patient appraisal within clinical practice [9], obtaining two measurements (one for general obesity, and one for central obesity) does not entail any extra cost [14]. In light of the increasing prevalence of cardiometabolic diseases worldwide [40], effective methods that help to identify subjects at greatest risk are needed. Risk stratification utilising BMI and WHtR together may provide a simple, cost-effective and more accurate technique for predicting obesity-related cardiometabolic events. Earlier identification of individuals at risk could enable earlier targeted interventions or therapies, thus attenuating development of cardiovascular complications.

\begin{abstract}
Abbreviations
AUC: area under the curve; BMl: body mass index; $\mathrm{BP}$ : blood pressure; $\mathrm{Cl}$ : confidence interval; C3: complement component 3; CRP: c-reactive protein; GHQ general health questionnaire; $\mathrm{HbA}_{1}$ : glycated haemoglobin $\mathrm{A}_{1 c} ; \mathrm{HDL}-\mathrm{C}$ : high density lipoprotein cholesterol; IL-6: interleukin 6; IPAQ: international physical activity questionnaire; MetS: metabolic syndrome; OR: odds ratio; PAI-1: plasminogen activator inhibitor-1; ROC: receiver operating characteristic curve; Rx: prescription; TNF- $\alpha$ : tumour necrosis factor alpha; VAT: visceral adipose tissue; WBC: white blood cell; WC: waist circumference; WHtR: waist-height ratio.
\end{abstract}

\section{Authors' contributions}

SRM conceived of the study and performed the statistical analysis. SRM, IJP and CMP drafted the final manuscript. All authors read and approved the final manuscript.

\section{Acknowledgements}

This work was supported by a research grant from the Irish Health Research Board (reference HRC/2007/13). The funders had no role in the study design, data collection and analysis, decision to publish or preparation of the manuscript.

\section{Compliance with ethical guidelines}

\section{Competing interests}

The authors declare that they have no competing interests.

Received: 27 March 2015 Accepted: 25 August 2015

Published online: 07 September 2015

\section{References}

1. Guh D, Zhang W, Bansback N, Amarsi Z, Birmingham CL, Anis A. The incidence of co-morbidities related to obesity and overweight: a systematic review and meta-analysis. BMC Public Health. 2009;9(1):88.

2. Phillips CM, Perry IJ. Does inflammation determine metabolic health status in obese and nonobese adults? J Clin Endocrinol Metab. 2013;98(10):E1610-9.
3. Grundy SM, Benjamin IJ, Burke GL, Chait A, Eckel RH, Howard BV, et al. Diabetes and cardiovascular disease a statement for healthcare professionals from the American Heart Association. Circulation. 1999;100(10):1134-46.

4. Connor JM, Millar SR, Buckley CM, Kearney PM, Perry IJ. The prevalence and determinants of undiagnosed and diagnosed type 2 diabetes in middle-aged Irish adults. PLoS One. 2013;8(11):e80504.

5. Collaboration PS. Body-mass index and cause-specific mortality in 900,000 adults: collaborative analyses of 57 prospective studies. Lancet. 2009:373(9669):1083-96.

6. Gómez-Ambrosi J, Silva C, Galofré J, Escalada J, Santos S, Millán D, et al. Body mass index classification misses subjects with increased cardiometabolic risk factors related to elevated adiposity. Int J Obes. 2011;36(2):286-94.

7. Curtis JP, Selter JG, Wang Y, Rathore SS, Jovin IS, Jadbabaie F, et al. The obesity paradox: body mass index and outcomes in patients with heart failure. Arch Intern Med. 2005;165(1):55.

8. Arsenault BJ, Després JP, Boekholdt SM. Hypertriglyceridemic waist: missing piece of the global cardiovascular risk assessment puzzle? Clin Lipidol. 2011;6(6):639-51.

9. Klein S, Allison DB, Heymsfield SB, Kelley DE, Leibel RL, Nonas C, et al. Waist circumference and cardiometabolic risk: a consensus statement from shaping America's health: Association for Weight Management and Obesity Prevention; NAASO, the Obesity Society; the American Society for Nutrition; and the American Diabetes Association. Obesity. 2012;15(5):1061-7.

10. Organization $\mathrm{WH}$. Waist circumference and waist-hip ratio: report of a WHO expert consultation. Geneva: Switzerland; 2008. p. 8-11.

11. Millar SR, Perry IJ, Phillips CM. Surrogate measures of adiposity and cardiometabolic risk - why the uncertainty? A Review of recent metaanalytic studies. J Diabetes Metab. 2013;S11:004. doi:10.4172/2155-6156 S11-004.

12. Ashwell M, Gibson S. Waist to height ratio is a simple and effective obesity screening tool for cardiovascular risk factors: analysis of data from the British National Diet and Nutrition Survey of adults aged 19-64 years. Obes Facts. 2009;2(2):97-103.

13. Ashwell M, Hsieh SD. Six reasons why the waist-to-height ratio is a rapid and effective global indicator for health risks of obesity and how its use could simplify the international public health message on obesity. Int J Food Sci Nutr. 2005;56(5):303-7.

14. Can AS. Body mass index, waist-to-height ratio, cardiometabolic risk factors and diseases in a new obesity classification proposal. Open Obes J. 2011;3:55-61.

15. Ashwell M, Gunn P, Gibson S. Waist-to-height ratio is a better screening tool than waist circumference and BMI for adult cardiometabolic risk factors: systematic review and meta-analysis. Obes Rev. 2012;13(3):275-86.

16. Savva SC, Lamnisos D, Kafatos AG. Predicting cardiometabolic risk: waist-to-height ratio or BMI. A meta-analysis. Diabetes Metab Syndr Obes Target Ther. 2013;6:403-19.

17. Kodama S, Horikawa C, Fujihara K, Heianza Y, Hirasawa R, Yachi Y, et al. Comparisons of the strength of associations with future type 2 diabetes risk among anthropometric obesity indicators, including waist-to-height ratio: a meta-analysis. Am J Epidemiol. 2012;176(11):959-69.

18. Caballero B. The global epidemic of obesity: an overview. Epidemiol Rev. 2007;29(1):1-5.

19. Kearney PM, Harrington JM, MC Carthy JM, Fitzgerald AP, Perry IJ. cohort profile: the cork and kerry diabetes and heart disease study. Int J Epidemiol. 2013;42(5):1253-62. doi:10.1093/ije/dys131.

20. Booth ML, Ainsworth BE, Pratt M, Ekelund U, Yngve A, Sallis JF, et al. International physical activity questionnaire: 12-country reliability and validity. Med Sci Sports Exerc. 2003;195(9131/03):1381-3508.

21. Grundy SM, Brewer HB Jr, Cleeman JI, Smith SC Jr, Lenfant C. Definition of metabolic syndrome report of the National Heart, Lung, and Blood Institute/American Heart Association Conference on scientific issues related to definition. Circulation. 2004;109(3):433-8.

22. Matthews D, Hosker J, Rudenski A, Naylor B, Treacher D, Turner R. Homeostasis model assessment: insulin resistance and $\beta$-cell function from fasting plasma glucose and insulin concentrations in man. Diabetologia. 1985;28(7):412-9.

23. Association AD. Diagnosis and classification of diabetes mellitus. Diabetes Care. 2013;36(Suppl 1):S67-74 
24. Phillips CM. Metabolically healthy obesity: definitions, determinants and clinical implications. Reviews in Endocrine and Metabolic Disorders. 2013;14(3):219-27.

25. Flegal KM, Kit BK, Orpana H, Graubard BI. Association of All-cause mortality with overweight and obesity using standard body mass index categories. Syst Rev Meta-Anal JAMA. 2013;309(1):71-82.

26. Shea JL, Randell EW, Sun G. The prevalence of metabolically healthy obese subjects defined by BMI and Dual-energy X-ray absorptiometry Obesity. 2011;19(3):624-30.

27. Chan JM, Rimm EB, Colditz GA, Stampfer MJ, Willett WC. Obesity, fat distribution, and weight gain as risk factors for clinical diabetes in men. Diabetes Care. 1994;17(9):961-9.

28. Carey VJ, Walters EE, Colditz GA, Solomon CG, Willet WC, Rosner BA, et al. body fat distribution and risk of non-insulin-dependent diabetes mellitus in women The Nurses' Health Study. Am J Epidemiol. 1997;145(7):614-9.

29. Kabir M, Catalano KJ, Ananthnarayan S, Kim SP, Van Citters GW, Dea MK, et al. Molecular evidence supporting the portal theory: a causative link between visceral adiposity and hepatic insulin resistance. Am J Physiol Endocrinol Metab. 2005;288(2):E454-61.

30. Millar SR, Perry IJ, Broeck JVD, Phillips CM. Optimal central obesity measurement site for assessing cardiometabolic and type 2 diabetes risk in middle-aged adults. PLoS One. 2015;10(6):e0129088. doi:10.1371/journal. pone.0129088.

31. McCarron P, Okasha M, McEwen J, Smith GD. Height in young adulthood and risk of death from cardiorespiratory disease: a prospective study of male former students of Glasgow University, Scotland. Am J Epidemiol. 2002;155(8):683-7.

32. Engeland A, Bjørge T, Selmer RM, Tverdal A. Height and body mass index in relation to total mortality. Epidemiology. 2003;14(3):293-9.
33. Alberti K, Zimmet P, Shaw J. Metabolic syndrome-a new world-wide definition. A Consensus Statement from the International Diabetes Federation. Diabet Med. 2006;23(5):469-80.

34. Stern MP, Williams K, González-Villalpando C, Hunt KJ, Haffner SM. Does the metabolic syndrome improve identification of individuals at risk of type 2 diabetes and/or cardiovascular disease? Diabetes Care. 2004;27(11):2676-81.

35. Bosy-Westphal A, Booke C-A, Blöcker T, Kossel E, Goele K, Later W, et al Measurement site for waist circumference affects its accuracy as an index of visceral and abdominal subcutaneous fat in a Caucasian population. J Nutr. 2010;140(5):954-61.

36. Phillips CM, Tierney AC, Perez-Martinez P, Defoort C, Blaak EE, Gjelstad $I M$, et al. Obesity and body fat classification in the metabolic syndrome: impact on cardiometabolic risk metabotype. Obesity. 2013;21(1):E154-61.

37. Ashwell M. Plea for simplicity: use of waist-to-height ratio as a primary screening tool to assess cardiometabolic risk. Clin Obes. 2012;2(1-2):3-5.

38. Standardization WECoB, Organization WH. Physical status: the use and interpretation of anthropometry: report of a WHO Expert Committee. World Health Organization; 1995.

39. Cronin S, Berger S, Ding J, Schymick JC, Washecka N, Hernandez DG, et al. A genome-wide association study of sporadic ALS in a homogenous Irish population. Hum Mol Genet. 2008;17(5):768-74.

40. Wild S, Roglic G, Green A, Sicree R, King H. Global prevalence of diabetes estimates for the year 2000 and projections for 2030. Diabetes Care. 2004;27(5):1047-53.

\section{Submit your next manuscript to BioMed Central and take full advantage of:}

- Convenient online submission

- Thorough peer review

- No space constraints or color figure charges

- Immediate publication on acceptance

- Inclusion in PubMed, CAS, Scopus and Google Scholar

- Research which is freely available for redistribution

Submit your manuscript at

www.biomedcentral.com/submit

C BioMed Central 